\title{
Common Architecture for Simulation of Critical Infrastructure Interdependencies in Emergency Situations
}

\author{
Georgi Kirov, Plamena Zlateva, and Dimiter Velev
}

\begin{abstract}
The paper discusses the main issues involved in a simulation of complex interdependent systems, which are a part of the Critical Infrastructure (CI). The purpose is to present a common architecture for simulation of critical infrastructure interdependencies in emergency situations. An analysis of the interdependencies between critical infrastructure elements and explanations of the process of development HLA/RTI (High Level Architecture/Run Time Infrastructure) simulations for study of CI elements are carried out. The approach provides a possibility of performing an assessment of the elements of the CI and their interdependencies affected by emergency situations as natural disasters.
\end{abstract}

Index Terms-Critical infrastructure, HLA/RTI, distributed simulations, emergency situations, natural disasters.

\section{INTRODUCTION}

Critical Infrastructure (CI) elements comprise a lot of basic facilities, services, information systems, and communication networks needed for the functioning of a community or society. People are becoming more and more dependent from these resources and assets due to the critical operations and infrastructures they support [1]. However, the infrastructure objects are vulnerable to a variety of disruptions, ranging from mild (short-term power outage, disk drive failure) to severe (natural disaster, equipment destruction, fire). Moreover, the infrastructure elements have complex relationships and interdependencies that cross infrastructure boundaries. This fact increases the spectrum of the threads. It raises the question for effective emergency management that considers infrastructure interdependencies and vulnerability so that the appropriate control can be put into place to either prevent incidents from happenings or to limit the effect of an incident [2]. In addition to this, the complex interdependencies between the components resulting in the mutual provision of services and use of common communications network, these systems cannot be studied directly. For example, it is not possible to directly test the effect of controlled shut-down procedures for segments of the power grid, the spread of a computer virus, or the spread of a disease vector under different vaccination options without having potentially drastic economic, safety or health impacts. For infrastructure networks, replicating all or even a part of the physical infrastructure may be prohibitively expensive. In both these cases, computational modeling and

Manuscript received December 9, 2015; revised March 23, 2016. This work was supported in part by the Bulgarian National Science Fund for the support under the Grant № DFNI-I02/ 15 from 2014.

The authors are with the ISER, Bulgarian Academy of Sciences, Sofia 1113, Bl. 2, Bulgaria (e-mail: g_tk@abv.bg, plamzlateva@abv.bg, gvelev@unwe.bg). simulation provides a safe and cost-effective alternative that can help enormously in developing needed understanding. The above-mentioned considerations show that the CI protection has to be a purpose not only of common strategies but also this problem has to be examined on a higher level concerning the national and international security [1]. There is a growing need to analyze and better understand the interdependencies that cross multiple infrastructure sectors and can induce potentially cascading effects [2]. The modeling and analysis of interdependencies between CI elements is a relatively new and very important field of study. A number of simulation models have been developed and more are being developed for studying individual aspect of infrastructure elements [3]. The value of these models decreases because they do not consider all aspects of a disaster. The simulation models addressing different aspects of an emergency situation need to be integrated in a common framework to provide the whole picture of a situation to planners, trainers, and responders [4], [5]. Distributed simulation technologies are a paradigm to model dynamic, heterogeneous, and spatial distributed systems. They not only aim at speeding up simulations, but also serve as strategic technologies for linking simulation components of various types [6]. Distributed technologies can run with different components installed on different computers linked via a local network so as to accelerate the execution time of the simulation [7].

The purpose of the paper is to present a common architecture for simulation of critical infrastructure interdependencies in emergency situations. An analysis of the interdependencies between critical infrastructure elements and explanations of the process of development HLA/RTI (High Level Architecture / Run Time Infrastructure) simulations for study of CI elements are carried out. This approach will provide a possibility of performing an assessment of the elements of the $\mathrm{CI}$ and their interdependencies affected by emergency situations as natural disasters.

\section{INTERDEPENDENCIES AND CASCADING EFFECTS}

The interdependencies and cascading effects between the infrastructure objects are analyzed. According to one of the most widespread definition, interdependency is a bidirectional relationship between infrastructure objects through which the state of each infrastructure object is influenced by the state of the other. The interrelationship among infrastructure objects is a precondition for cascading effects. The cascading effects can occur when an infrastructure object disruption spreads beyond itself to cause 
appreciable impact on other infrastructure objects, which in turn cause more effects on still other infrastructure objects. The consequences of the cascading effects due to an infrastructure failure can range from the mild to the catastrophic. For example, interdependencies exist between different sectors: national energy, water supply system, transport, telecommunication, and emergency services. A power outage can cause an effect on water supply system. The impact of the disruption may not stop at this level. It may go on to adversely affect other critical infrastructure objects (Fig. 1).

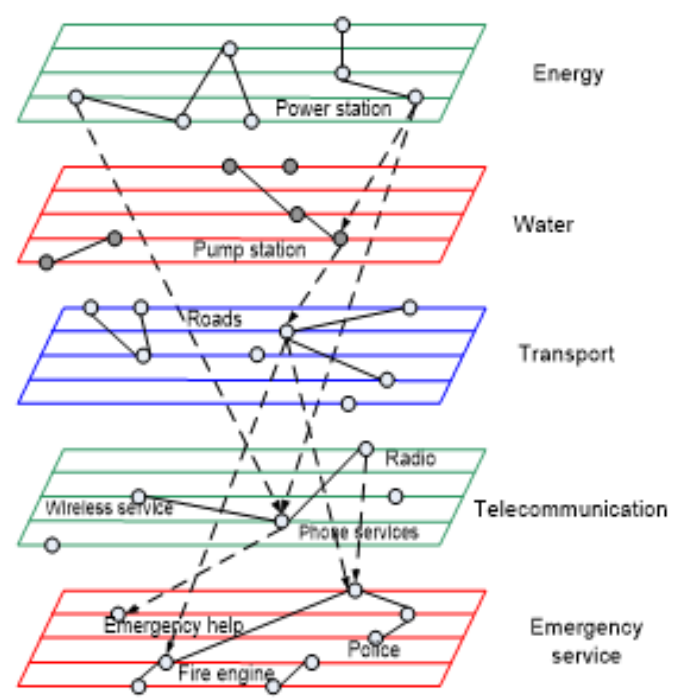

Fig. 1. Critical infrastructure interdependencies.

Given the current state of shrinking budgets and growing number of threats, it is obvious to almost any observer in the field of the emergency management that there is a critical need for a system analysis of the CI interdependencies. According to the system analysis there are four classes of interdependencies: physical, cyber, geographic, logical [8], [9]:

- Physical interdependency - two infrastructure objects are physically interdependent if the state of each depends upon the material output of the other. A requirement for this interdependency is a physical reliance on material flow from one infrastructure to another.

- Cyber interdependency - two infrastructure objects are cyber interdependent if the state of the one depends on information transmitted through the information system of the other. This is relatively new type of interdependency which basic element is information transfer between infrastructure objects. For example, a lot of infrastructure objects use the SCADA systems for control and analysis. Therefore, the infrastructure objects have an information dependency from the SCADA systems.

- Geographic interdependency - two infrastructure objects are geographically interdependent if a local environmental event can change the state in the two objects. The main requirement for this type of interdependency is the existence of a physical proximity. A fire may affect and disrupt all the infrastructure objects located in the area. It is important to notice, that geographic interdependency exists not due to physical connections between infrastructure objects; rather, it arises from the influence the event exerts on all infrastructure objects simultaneously.

- Logical interdependency - two infrastructures are logically interdependent if the state of each depends upon the state of the other via some mechanism that is not a physical, cyber, or geographic connection. This type of interdependency shows infrastructure components effects on social factors such as public opinion and cultural issues. For example, various regulatory mechanisms can give rise to logical linkage among two or more infrastructure objects [10].

Modeling and simulation of the interdependencies between the critical infrastructure objects is a complex, multidisciplinary process, which implies the work of the different specialists - mathematicians, programmers, physicists, and so on. The interdependency formalization is basic requirement for applicability of the computer simulation approach for analysis of the infrastructure interdependencies. Therefore, the above-mentioned considerations raise questions about the approaches for the infrastructure interdependencies. Analytical approaches study the problem based on over-simplified assumptions. This means the final results to be biased towards interdependencies under ideal conditions. The inaccuracy of analytical models focuses the expert attention on simulation to obtain correct results.

\section{Choice of A Simulation TeChNOlogy FOR ANALYSIS OF THE CRITICAL INFRASTRUCTURES}

Concerning the characteristics of the infrastructure interdependencies and the results from the system analysis the main requirements for applicability of a simulation technology to the field are defined. The parameters given in Table I can be used as criteria for a choice of a simulation technology.

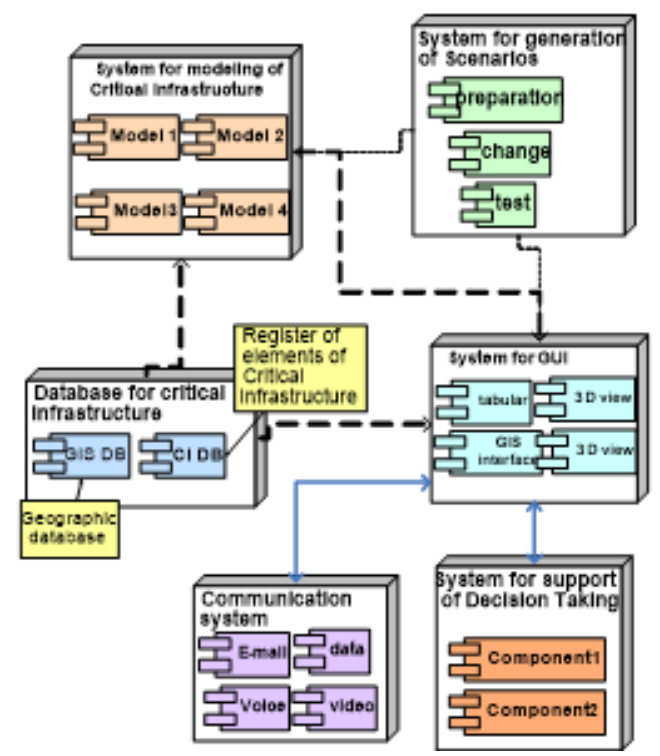

Fig. 2. Components of the simulation system.

The analysis of the infrastructure interdependencies is a complex process that makes the use of traditional simulation 
approaches inefficiency. For example, traditional simulators do not attempt to address the all aspect of an incident response. Therefore, they cannot simulate the infrastructure interdependencies and cascading effects. It results in critical need for a new and innovative simulation approach that allows an information exchange and time synchronization between infrastructure objects. The major difference between traditional simulation systems and proposed one is certainly that contemporary simulation applications not only require the exchange of data during their runtime but also require the exchange of synchronization information regarding their advancement of simulation time.

Fig. 2 shows a common architecture, which matches the requirements defined in Table I. It identifies which required systems support the operational view requirements. It translates the required degree of interoperability into a set of needed system capabilities and compares current implementations with needed capabilities.

TABLE I: REQUIREMENTS FOR APPLICABILITY OF SIMULATION TECHNOLOGY

\begin{tabular}{|c|c|}
\hline Parameters & Parameter analysis - relevance to computer simulation \\
\hline $\begin{array}{l}\text { Interoperability } \\
\text { and reusability }\end{array}$ & $\begin{array}{l}\text { It means that simulation models can be reused in different } \\
\text { simulation scenarios and applications. Closely related to } \\
\text { reusability is the property of interoperability, which means } \\
\text { that the reusable component simulations can be combined } \\
\text { with other components without the need for re-coding. } \\
\text { Interoperability implies an ability to combine component } \\
\text { simulations on distributed computing platforms of different } \\
\text { types, often with real-time operation. }\end{array}$ \\
\hline Tame scale & $\begin{array}{l}\text { The critical infrastructure has a very important characteristic } \\
\text { - dynamics. The dynamics investigation is hard task because } \\
\text { it varies from milliseconds to decades. The interdependency } \\
\text { analysis requires the using of the simulation technology, } \\
\text { which can control the time. }\end{array}$ \\
\hline Geographic Scale & $\begin{array}{l}\text { Simulation technology has to support different geographic } \\
\text { scale. It allows an investigation of different scenarios in } \\
\text { respect to geographic situation of the infrastructure objects. } \\
\text { Varying from cities to national level. The ability for scaling } \\
\text { increases the quality of interdependency analysis - } \\
\text { resolution, information exchange level, and so on. }\end{array}$ \\
\hline Cascading effects & $\begin{array}{l}\text { The simulation technology has to simulate the cascading } \\
\text { effect, i.e. it has to integrate different simulation models in a } \\
\text { common framework to provide the whole picture of a } \\
\text { disaster. }\end{array}$ \\
\hline
\end{tabular}

\section{HLA SimUlATION ARCHITECTURE FOR ANALYSIS OF THE CRITICAL INFRASTRUCTURES}

The proposed simulation architecture allows communication between simulation models based on High Level Architecture/Run Time Infrastructure (HLA/RTI) standard for information exchange. This technology can address the infrastructure interdependencies between multiple independent aspects of emergency situation and simulates the overall effect on the vital infrastructure objects. HLA is the IEEE standard for software architecture of interoperable distributed simulations. HLA aims to establish a common high-level simulation architecture to facilitate the interoperability of all types of models and simulations among themselves, as well as to facilitate the reuse of modeling and simulation (M\&S) components [11]. HLA allows experts to combine computer simulations into larger simulation. For instance, the experts might want to combine simulations of critical infrastructures in several different regions of the country. HLA can extend simulation later by adding new models or simulations, for example new models of infrastructures.

The relationships of HLA components are shown in Fig. 3. The conceptual model includes simulators, data collectors, passive viewers, and lives surrogates simulations. They have a single point of attachment to the RTI. The federate might be a surrogate for human interactions. In this role it can effects the states of the remaining federates in simulation [12].

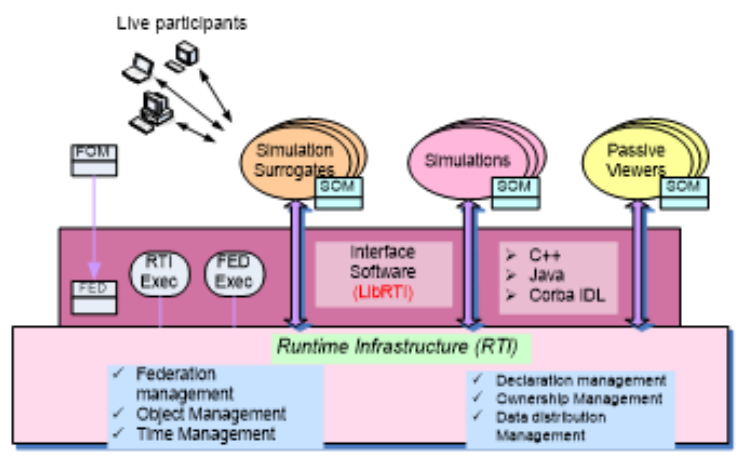

Fig. 3. HLA concepts.

Fig. 4 presents architecture for distributed simulation that provides communication infrastructure for the models' coordination and time synchronization. The proposed architecture is intended to provide a generic platform that allows communication between simulation models based on HLA/RTI standard for information [13], [14]. The benefit of this approach is a possibility of doing an assessment of the elements of the CI affected by an emergency situation [15], [16]:

- Simulators - Different simulators are integrated in the simulation environment to simulate the disaster. These modules are used to model a wide range of activities concerning disaster and its impact on the critical infrastructure. For example, simulators model the first and secondary impact of the disaster, the action of response services such government agencies, fire, police, medical personal and other services and organization responsible for rescue activities. The simulators allow evaluating the complex impact of environmental factors on the disaster impact.

- Distributed simulation environment-Models developed with different simulators can be integrated and communicate through common communication environment based on the HLA standard. It provides the communication functions and services that define a simulation interface specification, which permits a set of independently-developed simulations, to be brought together into a coordinated complex system. It means that different models can communicate using HLA/RTI services [16].

- Scenario emulator - This module provides the communication medium between simulators and visualization systems. It gathers the information from simulators and evaluates the status of all simulated objects. In the next step it sends the information as an input of the visualization.

- Database - It supports all information for a normal 
operation of the simulation. For example, terrain and city maps, GIS data, and information about the buildings and objects of the CI. In addition, the database contains a great number of files required by different simulators such as natural disaster scenario files.

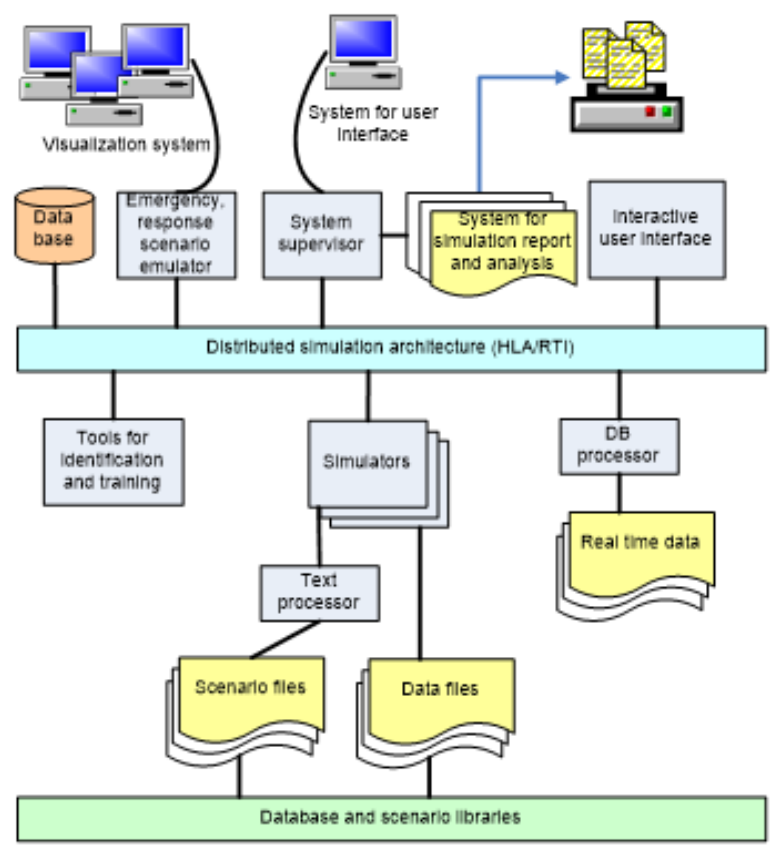

Fig. 4. HLA simulation architecture.

- Interactive user interface - This module is very important element from the simulation architecture. The software engineers use them for the synchronization and system settings of the complex simulation environment. Interactive user interface can be used to generate and modify different disaster scenarios. Furthermore, this tool is used for visualization of the scenario taking into account the results from the emulators [9].

- Tools for identification and training - The main purpose of this module is a training of the employees that are responsible for rescue activities and emergency management. They can test their degree of preparation and qualification using interaction with the simulation.

The proposed distributed simulation architecture can integrate the different simulation models in a common distributed framework. More important, this technology provides information exchange between them. It allows simulation of many aspects of the disaster and evaluating the overall effect on the critical infrastructure.

\section{A CASE STUdy: AN EXAMPLE OF INTEGRATED SIMULATION SYSTEM}

To verify the effectiveness of the new simulation technology, a case study was done on an integrated simulation of critical infrastructure interdependencies and their control mechanisms. The purpose of the integrated simulation is to observe how the critical infrastructure objects behave when unordinary events (for example, natural disaster) occur. The integrated simulation system is created from a set of models that are interconnected with each other. The proposed simulation system consists of several functional components [5], [16]:

- Simulation models: All simulated entities, such as different infrastructure elements or threats, are referred to as simulation models. It includes models of infrastructure objects, data collectors, and disasters. The simulation models consist of $\mathrm{C}++$ code that access communication services provided by the RTI communication environment. This mechanism allows communication between simulation models based on HLA standard through RTI infrastructure. The communication between infrastructure objects in the integrated simulation is based on a common object model. It contains exchange data created by the developer that shows the relationships between models. Hence, the common object model defines object classes, their attributes and interaction classes that are commonly used and exchanged among models in the simulation.

- Viewer application: The viewer is developed to provide an integrated display environment (Fig. 5). It can act as a passive recipient and display simulation data from the rest of simulation system. The viewer is an important part of the simulation system because it provides analysis tools and playback capabilities. The viewer communicates with the simulation models over TCP/IP protocol that allows different models to reside on separate computers [5].

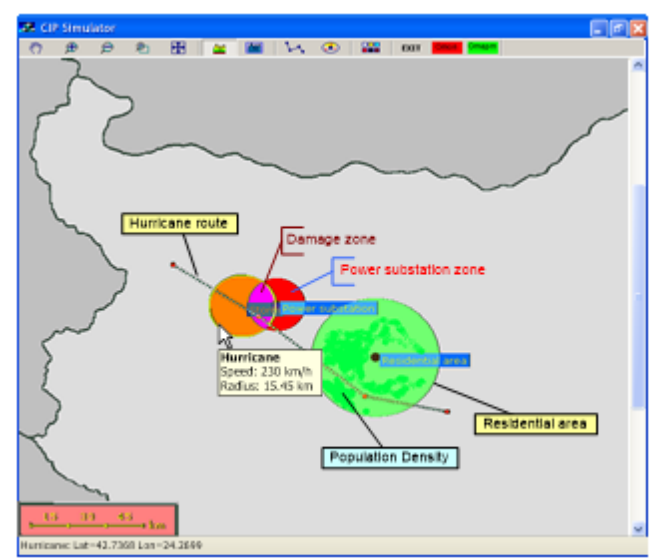

Fig. 5. Viewer application for hurricane simulation

The scenario for this case study attempts to incorporate realism and flexibility. The aim of the example is to evaluate the impact of a hurricane and potentially cascading effects, to test the effectiveness of the emergency response plans, for helping train response personnel, and for vulnerability analysis. As a result of repeated execution of simulation, data is collected and analyzed, and the results are documented. The simulation results are presented in Table I. The table displays simulation time and state variables of the interdependent models exchanged through RTI environment. The row of the table represents the time series of a state variable. The column represents the set of the simulated variables. The state of the variables at time $t$ depends only on the states before $t$. The simulation results can be used for an analysis and assessment of the cascading effects and improving critical infrastructure protection in cases of emergency situations, as natural disasters. 
TABLE II: SiMULATION RESULTS

\begin{tabular}{|c|c|c|c|c|c|c|c|}
\hline \multicolumn{8}{|c|}{ Space-time graph for disaster simulation } \\
\hline \multirow{4}{*}{ Hurricane } & Latitude & 43.2299 & 43.2269 & 43.0365 & 43.9603 & 42.7368 & 42.5868 \\
\hline & Longitude & 23.3271 & 23.3336 & 23.7375 & 23.8540 & 24.2699 & 24.5196 \\
\hline & Speed $[\mathrm{km} / \mathrm{h}]$ & 190 & 190 & 220 & 220 & 220 & 220 \\
\hline & Latitude & 43.0295 & 43.0295 & 43.0295 & 43.0295 & 43.0295 & 43.0295 \\
\hline \multirow[t]{4}{*}{ Power sub-station } & Longitude & 24.0012 & 24.0012 & 24.0012 & 24.0012 & 24.0012 & 24.0012 \\
\hline & Damages [\%] & - & - & 15 & 30 & 35 & 35 \\
\hline & Latitude & 42.7514 & 42.7514 & 42.7514 & 42.7514 & 42.7514 & 42.7514 \\
\hline & Longitude & 24.6549 & 24.6549 & 24.6549 & 24.6549 & 24.6549 & 24.6549 \\
\hline \multirow[t]{3}{*}{ Residential area } & People in disaster area [\%] & - & - & - & 2 & 23 & 58 \\
\hline & Electricity blackout [\%] & - & - & - & 32 & 43 & 56 \\
\hline & Damaged buildings [\%] & - & - & - & - & 16 & 23 \\
\hline \multicolumn{2}{|c|}{ Simulation time [sec] } & 300 & 310 & 880 & 1120 & 1750 & 2160 \\
\hline
\end{tabular}

\section{CONCLUSION}

The proposed simulation architecture is based on a distributed framework that can be rapidly implemented with interoperability standards for the modeling and simulation. Together, the framework and interoperability standards can significantly increase the use of modeling and simulation for disaster management. In turn, it will help improve the emergency management capabilities. This approach provides a possibility of doing an assessment of the elements of the CI and their interdependencies affected by an emergency situation due to natural disasters. The main concept relies on the idea of providing a structural methodology and tools for the use of the methods to design, develop, and implement the HLA system, and obtain the interoperation and reuse of the simulation models. The potential advantages of the proposed architecture are evident: increased flexibility, building on existing software and communications standards, and maximisation of the use of existing simulation models.

\section{ACKNOWLEDGMENT}

The authors express their gratitude to the Bulgarian National Science Fund for the partial financial support under the Grant № DFNI-I02/15 from 12.12.2014, titled "Information System for Integrated Risk Assessment from Natural Disasters".

\section{REFERENCES}

[1] P. Edwards, "Millennial reflections on computers as infrastructure," History and Technology, vol. 15, pp. 7-29, 1998.

[2] M. S wanson, A. Wohl, L. Pope, T. Grance, J. Hash, and R. Thomas, "Contingency planning guide for information technology systems," NIST Special Publication, 2002.

[3] S. Jain and C. McLean, "A framework for modelling and simulation of emergency response," in Proc. 35th Conference on Winter Simulation: Driving Innovation, New Orleans, Louisiana, 2003, pp. 1068-1076.

[4] S. Jain and C. McLean, Modelling and Simulation of Emergency Response: Workshop Report, Relevant Standards and Tools, National Institute of Standards and Technology Internal Report, NISTIR-7071, 2003.

[5] G. Kirov and V. Stoyanov, "Software architecture for implementation of complex simulation systems," Cybernetics and Information Technologies, vol. 8, no. 4, pp. 57-68, 2008.

[6] D. Chena, S. Turner, W. Cai, and M. Xiong, "A decoupled federate architecture for high level architecture-based distributed simulation," $J$. Parallel Distrib. Comput., vol. 68, pp. 1487-1503, 2008.

[7] A. Tolk, "HLA-OMT versus traditional data and object modeling," in Proc. Command and Control Research and Technology Symposium, Annapolis, Maryland, 2001.
[8] S. Rinaldi, J. Peerenboom, and T. Kelly, "Identifying, understanding and analyzing critical infrastructure interdependencies," IEEE Control Systems Magazine, pp.11-25, 2001

[9] P. Pederson, D. Dudenhoeffer, S. Hartley, and M. Permann, "Critical infrastructure interdependency modeling: A survey of U.S. and international research," The Technical Support Working Group Under Work for others Agreement 05734, 2006.

[10] S. Rinaldi, "Modeling and simulating critical infrastructures and their interdependencies," in Proc. 37th Hawaii International Conference on System Sciences, IEEE, 2004.

[11] IEEE Standard for Modeling and Simulation (M\&S), IEEE Std 1516.2-2000, 2010.

[12] F. Kuhl, R. Weatherly, and J. Dahmann, Creating Computer Simulation Systems: An Introduction to the High Level Architecture, Prentice Hall PTR, 1999.

[13] J. Kim and T. Kim, "Hierarchical HLA: Mapping hierarchical model structure into hierarchical federation," in Proc. M\&S-MTSA '06, Ottawa, Canada, 2006, pp. 75-80.

[14] The HLA. [Online]. Available: http://www.dmso.mil/public/transition/hla

[15] S. Jain and C. McLean. An architecture for integrated modeling and simulation for emergency response. [Online]. Available: http://www.mel.nist.gov/msidlibrary

[16] G. Kirov, "Integration of simulation models of the elements of the critical infrastructure in common simulation environment," in Proc. Scientific Support of the Transformation in Security Sector Conference, CNSDR-BAS, Sofia, 2006, pp.143-159.

Georgi Kirov is currently associate professor at the Institute of System Engineering and Robotics at the Bulgarian Academy of Sciences, Sofia, Bulgaria. He holds M.Sc. his degrees in computer systems from the Sofia Technical University and a Ph.D. degree in soft computing technologies from the Institute of Computer and Communication Systems. His main areas of academic and research interest are distributed information technologies, computer simulation, and risk management.

Plamena Zlateva is currently associate professor at the Institute of System Engineering and Robotics at the Bulgarian Academy of Sciences, Sofia, Bulgaria. She hods her M.Sc. degrees in applied mathematics from the Sofia Technical University and in economics from the Sofia University St. Kl. Ohridski, and a Ph.D. degree in manufacturing automation from the Institute of Conrol and System Research - BAS. Her main areas of academic and research interest are control theory, mathematical modeling and risk management.

Dimiter Velev is a professor in the Department of Information Technologies and Communications at the University of National and World Economy, Sofia, Bulgaria. He holds his M.Sc. degree in electroengineering from the Sofia Technical University, Bulgaria and a Ph.D. degree in engineering sciences from the Institute of Modeling Problems in Power Engineering at the National Academy of Sciences of Ukraine, Kiev, Ukraine. His main areas of academic and research interest are internet-based business systems modeling and development, service oriented architectures, online social networks, cloud computing, web applications development and programming. 